\title{
DAS VÖLKERWANDERUNGSZEITLICHE KINDERSKELETT von Schwarzenbach-Burg, Bezirk Wr. Neustadt, NiederösterReich, Mit KÜNSTLICH DEFORMIERTEM SCHÄDEL
}

\author{
Karin WILTSCHKE-SCHROTTA, Doris PANY, Wien
}

\section{Einleitung}

Im Jahre 1998 wurde an der höchsten Stelle des in der späten Eisenzeit massiv befestigten Hügels Burg in Schwarzenbach (Niederösterreich) im Vorfeld der Errichtung eines Aussichts- und Museumsturms im Auftrag des Bundesdenkmalamtes eine archäologische Grabung durch die Universität Wien unter der Leitung von Dr. Wolfgang Neubauer vorgenommen. Nach der maschinellen Entfernung einer illegal errichteten Betonplatte kamen die Reste eines Wachturmes und einer Mannschaftsbaracke aus dem zweiten Weltkrieg zu Tage. Durch die Errichtung dieses Beobachtungspostens waren Schichten aus der späten Eisenzeit, der Urnenfelderzeit und dem Spätneolithikum zerstört worden. Für die aus Holz errichtete Baracke war im Zweiten Weltkrieg die prähistorische Schichtenfolge auf einer Fläche von $22 \mathrm{~m}^{2}$ bis auf den anstehenden Orthogneis und Marmor abgegraben und ein Fundamentgraben für die Barackenmauer ausgehoben worden. Beim Putzen der Unterkante dieser Störung wurde während der Grabung eine in den Felsen eingetiefte Grube sichtbar, an deren westlichem Rand ein Schädel erkennbar war. Die Grabgrube ist annähernd rechteckig, senkrecht in den Felsen gehauen, ist 0.5 $\mathrm{m}$ breit und $1.2 \mathrm{~m}$ lang. Ihrer Längsachse ist genau West-Ost orientiert und wurde an der topographisch höchsten Stelle des Hügels angelegt. Sie wird im Osten durch den Fundamentgraben der Nordmauer der Baracke gestört.

Das freigelegte Skelett eines Kindes war von einer Steinlage aus Marmorbruchsteinen mit 5-20 cm Durchmesser bedeckt. Die Bestattung war im Fußbereich durch den
Fundamentgraben der Barackenmauer gestört. Es ließen sich bei der Grabung keine Grabeinbauten oder Spuren einer Beraubung feststellen. Das Kind wurde mit dem Kopf Richtung Westen (Blick nach Norden) ohne Grabbeigaben gefunden ${ }^{1}$. Die Lage des Skeletts deutete auf eine Bestattung in leichter linksseitiger Hockerstellung hin. Nach Analyse des Grabungsfotos (Abb. 1) und der Planaufnahme kann aber auch eine Bestattung in Rückenlage mit sekundärer Verlagerung der Oberschenkelknochen angenommen werden, da die Unterschenkelreste parallel und in der Körperachse liegen.

Die geborgenen Skelettreste wurden zur Bearbeitung und Präparation an die Mitarbeiter der Abteilung für Anthropologie des Naturhistorischen Museums Wien übergeben. Die fragilen Knochenreste wurden für die Untersuchung vorsichtig gereinigt und sorgfältig präpariert. Die erst dadurch erkennbar gewordene künstliche Schädeldeformation veranlasste uns zur vorliegenden Fallpublikation. Das untersuchte Kinderskelett wurde nach Errichtung des Aussichts- und Museumsturmes in einer rekonstruierten Grabgrube an Ort und Stelle wieder bestattet.

\section{Datierung (Peter Stadler)}

Da keinerlei Grabbeigaben vorlagen wurde zur zeitlichen Einordnung des Grabfundes eine ${ }^{14} \mathrm{C}-\mathrm{Datierung}$ am Knochenmaterial durchgeführt (VERA 1666: 1635 35 BP). Dazu wurde eine Kollagenextraktion durchgeführt, weil nur der Kollagen-Kohlenstoff, nicht aber der Kohlenstoff im 


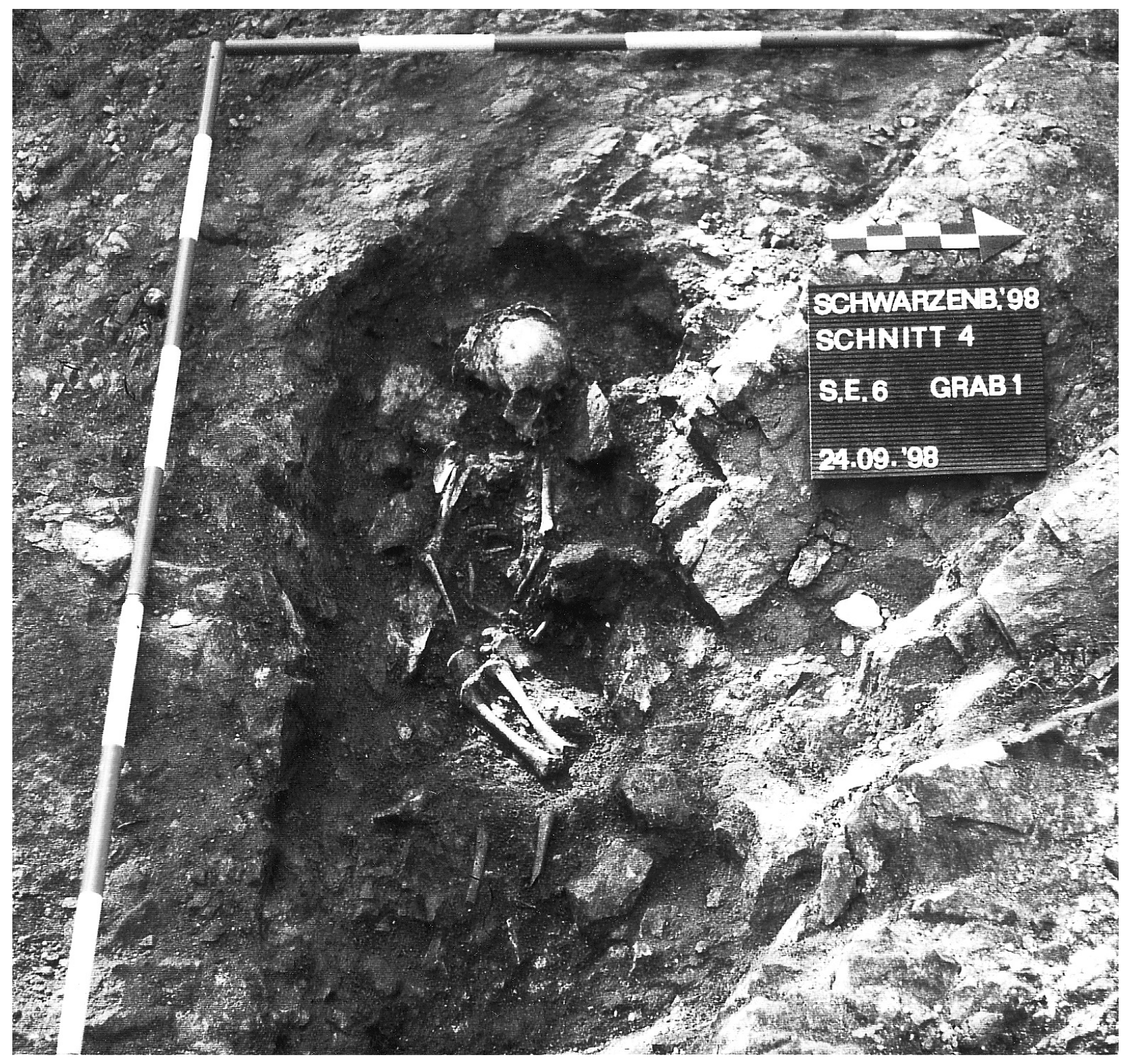

Abb. 1. Schwarzenbach-Burg, Grab 1, 1998.

$\mathrm{CaCO}_{3}$ des Knochens von einem späteren Kohlenstoffaustausch sicher und für eine Datierung geeignet ist. Das Ergebnis der Kalibration des Datierungsergebnisses ist in Abbildung 2 dargestellt.

Die Hauptlösung auf dem 1 Sigma-Niveau ist das Intervall von 380-440, daneben wären auch die anderen Intervalle möglich, diese sind aber nicht so wahrscheinlich. Somit liegt die wahrscheinlichste Datierung in der ersten Hälfte des 5. Jhs. nach Christus.

Das im Zeitraum von 380-440 n.Chr. verstorbene Kind stammt aus der Periode der Völkerwanderungszeit. Aufgrund der Datierung der Bestattung wäre eine gestreckte
Rückenlage zu erwarten anstelle der vorgefundenen Hockerbestattung. Die beobachtete künstliche Schädeldeformation war zu Beginn des 5. Jhs. vor allem zur Zeit der hunnischen Herrschaft verbreitet, sowohl bei den mongolischen Neuankömmlingen als auch bei den Ostgermanen des Donauraumes. In diesem zeitlichen Zusammenhang überrascht die Tatsache, dass keine Beigaben gefunden wurden. Die Verteilung der Steine der Grababdeckung deutet auf eine Beraubung im Brust- und Beinbereich hin. Die festgestellte sekundäre Verlagerung der Oberschenkelknochen könnte im Zuge einer Beraubung des Grabes erfolgt sein. 


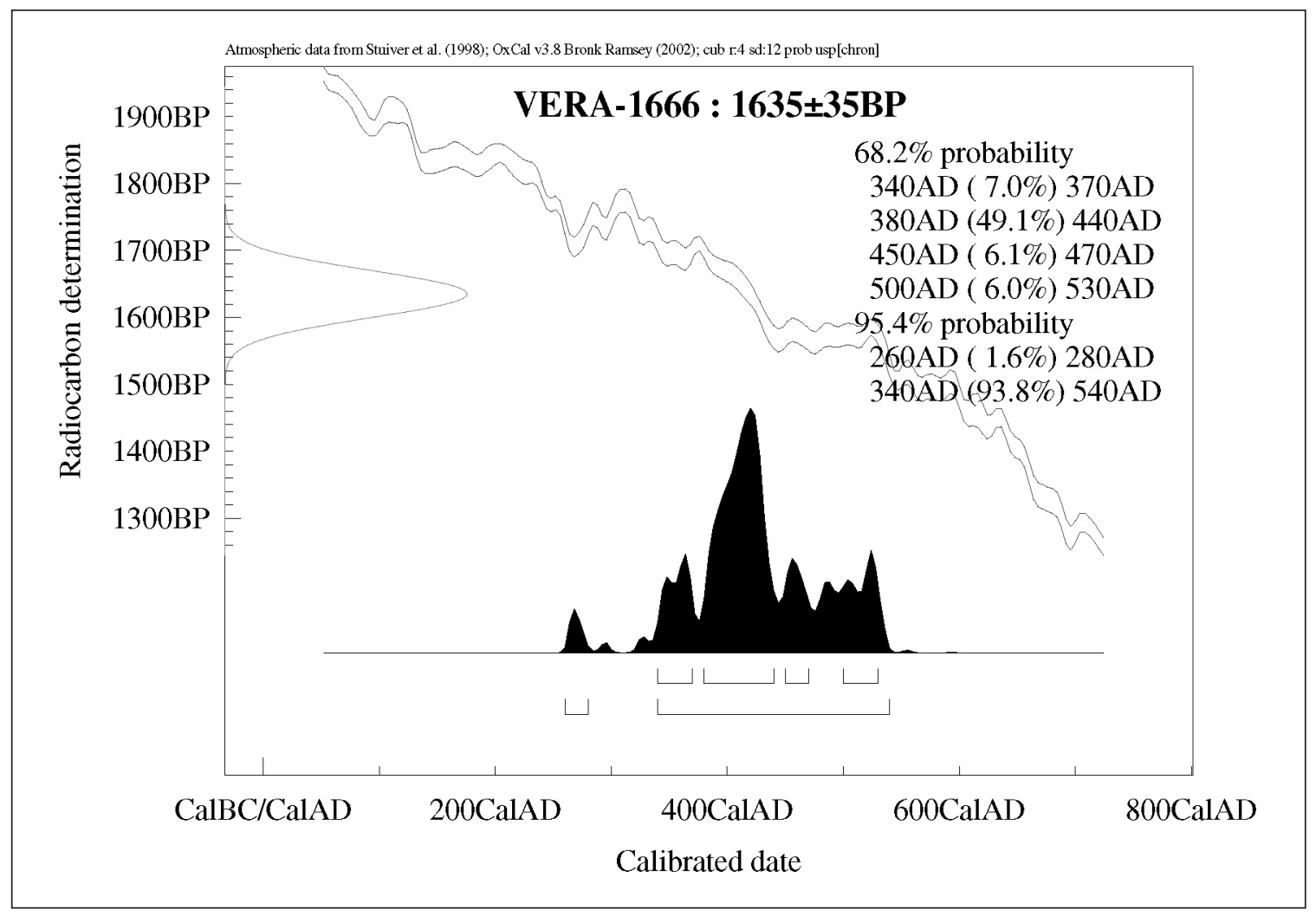

Abb. 2. Schwarzenbach-Burg, Ergebnisse der Kalibration mit Programm OxCal 3.8.

Die parallele Lage der Oberschenkelknochen, wegen der man während der Grabung von einer Hockerbestattung ausgegangen ist, könnte ein Hinweis darauf sein, dass eine Beraubung des Grabes kurz nach der Bestattung erfolgt ist. Die markante Lage des Grabes und die künstliche Schädeldeformation sprechen für eine besondere Bestattung, die ohne Grabbeigaben schwer vorstellbar ist.

\section{Anthropologischer Befund}

\section{Bestimmungsmethoden}

Das Sterbealter des Kindes wurde mittels der Diaphysenlänge $^{2}$ und des Entwicklungsstandes der Zahnanlagen ${ }^{3}$ be-

M. Stloukal, H. HanÁková, Die Länge der Längsknochen altslawischer Bevölkerungen unter besonderer Berücksichtigung von Wachstumsfragen, Homo 29, 1978, 53-69.

3 D. UbeLAKer, Human skeletal remains. Excavation, analysis, interpretation, Chicago1978. - C.F.A Moorrees, E.A. Fanning, E.E. Hunt, Age Formation by Stages for Ten Permanent Teeth, Journal of Dental Research 42, 1963, 1490-1502.

4 Zwentendorf Grab 503 Jahre, Zwentendorf Grab 112/1 5-7 Jahre. Die Schädel sind aus dem slawenzeitlichen Gräberfeld und stimmt. Für die Schädelumrisszeichnungen wurden genormte digitale Bilder im Photoshop 5. $5^{\mathrm{tm}}$ übereinander projiziert. Zum Vergleich mit nicht deformierten Kinderschädeln dienten die Schädel eines dreijährigen und eines fünfjährigen Kindes ${ }^{4}$ aus dem slawenzeitlichen Gräberfeld von Zwentendorf.

Die Körperhöhe des Kindes wurde nach A. Telkkä et al. ${ }^{5}$ geschätzt. Krankheitsbedingte Veränderungen am Skelett sind nach M. Schultz ${ }^{6}$ beurteilt worden.

\section{Erhaltung}

Von der Kinderbestattung sind ein stark verzogener, aus vielen Einzelteilen rekonstruierter Schädel und ein fast

sehr gut erhalten. Sie stammen aus der osteologischen Vergleichsammlung der Anthropologischen Abteilung, Inventarnummer 23.124 und 23.242

5 A. Telkk $\ddot{A}$, A. Palkoma, P. Virtama, Prediction of stature from radiographs of long bones in children, J. Forensic Sciences 7, 1962, 474-479.

6 M. Schultz, Paläopathologische Diagnostik. In: R. Knussmann (Hrsg.) Anthropologie - Handbuch der vergleichenden Biologie des Menschen. Band I, 1, Stuttgart 1988, 480-495. 


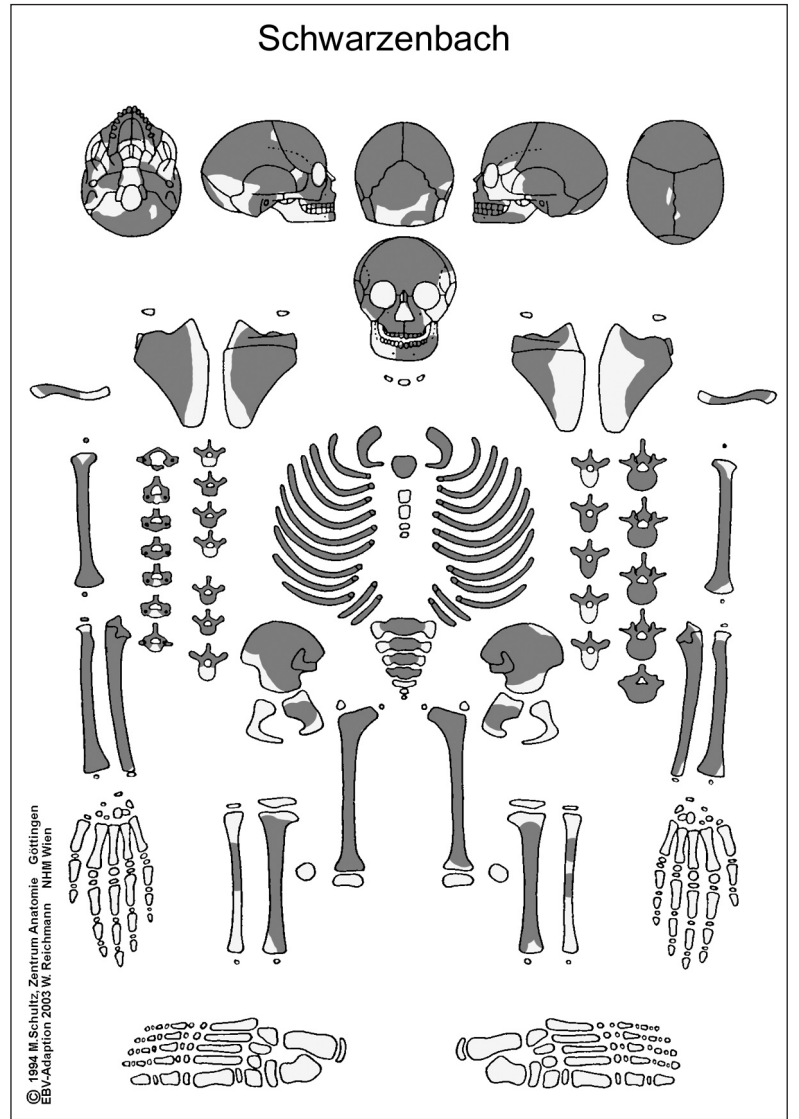

Abb. 3. Schwarzenbach-Burg, erhaltene Skelettknochen von Grab 1.

vollständiges postkraniales Skelett erhalten (Abb. 3). Der Gesichtsschädel ist nur teilweise vorhanden und der Unterkiefer ist stark beschädigt. Die Knochenoberfläche ist durchgehend leicht erodiert. Die Epiphysen der meisten Langknochen sowie die Hand- und Fußknochen sind nicht erhalten. Die Milchzähne des Unterkiefers sind fast vollständig, aber isoliert vorhanden.

\section{Befund des Gebisses}

Die Milchzähne (römische Ziffern) sind vollständig im Oberkiefer erhalten. Die Anlagen der ersten oberen Dauer-

\begin{tabular}{lcccccc|ccccc} 
& \multicolumn{6}{c|}{ rechts } & & \multicolumn{4}{|c}{ links } \\
& 6 & & & & & & & & & & \\
Oberkiefer & & V & IV & III & II & I & I & II & III & IV & V \\
\hline Unterkiefer & & V & & III & & & & II & III & IV & V \\
& 6 & & 4 & 3 & 2 & 1 & 2 & 3 & &
\end{tabular}

7 Ubelaker, s. Anm. 3.

Moorrees et al., s. Anm. 3.

Verwächst zwischen dem 1.-3. Lebensjahr. W.M. BAss, Human molaren (arabische Ziffern) sind rechts im Kiefer und links isoliert vorhanden. Aufgrund des schlechten Erhaltungszustandes des Unterkiefers liegen sowohl die Milchzähne als auch die Dauerzahnanlagen des Unterkiefers isoliert vor. Die Zahnabrasion ist gering, nur an den Schneidezähnen ist bereits eine dünne Dentinlinie erkennbar. Die Milchzähne weisen keine pathologischen Veränderungen wie z.B. Kariesbefall auf. Lediglich der linke obere erste Milchschneidezahn hat leichte Schmelzunregelmäßigkeiten an der bukkalen Zahnoberfläche. Diese deuten auf eine Anlageanomalie oder eine Wachstumsstörung hin. Als morphologische Variante sind an den zweiten Milchmolaren und an den ersten Dauermolaren des Oberkiefers große Tuberculi Carabelli zu erkennen (Abb. 4).

\section{Altersbestimmung anhand der Zahnentwicklung}

Der Mineralisationsgrad der isolierten Dauerzahnanlagen entspricht nach D. Ubelaker ${ }^{7}$ einem Entwicklungsalter von 4 Jahren +/- 12 Mon. Für die Altersbestimmung nach C.F.A. Moorrees et. al. ${ }^{8}$ wurde zusätzlich die Wurzelresorption der Milchzähne herangezogen und es wurde ein Sterbealter von etwa 3 Jahren festgestellt.

\section{Altersbestimmung anhand der Langknochenmaße}

Von den vorhandenen Langknochen wurde die größte Diaphysenlänge gemessen. Bei beschädigten oder fehlenden Enden wurde eine Längenschätzung gemacht und der Wert in Klammer angeführt (Tab. 1).

\section{Altersbestimmung anhand des Wachstumsfugenschlusses}

Die rechten und linken Wirbelbogenhälften sind bereits obliteriert ${ }^{9}$. Die Verschmelzung des Wirbelbogens mit dem Wirbelkörper hat noch nicht stattgefunden ${ }^{10}$.

\section{Geschätzte Körperhöhe}

Die Körperhöhe wurde aus der Länge des rechten Femur berechnet. Daraus ergibt sich für subadulte männliche Indi-

Osteology. A Laboratory and Field Manual, 3rd ed, Missouri Archaeological Society, Columbus, Missouri, 1987.

10 Verwächst zwischen dem 3.-7. Lebensjahr. BAss, s. Anm. 9. 
Tabelle 1. Langknochenmaße

\begin{tabular}{|l|l|l|}
\hline & Diaphysenlänge in $\mathrm{mm}$ & Sterbealter \\
\hline Humerus re/li & $(140)$ & 4 Jahre \\
\hline Ulna re/li & $(120)$ & 4 Jahre \\
\hline Radius re/li & $(110)$ & 4 Jahre \\
\hline Femur li & $(185)$ & 4 Jahre \\
\hline Femur re & 188 & 4 Jahre \\
\hline Tibia re/li & $(140)$ & 3 Jahre \\
\hline
\end{tabular}

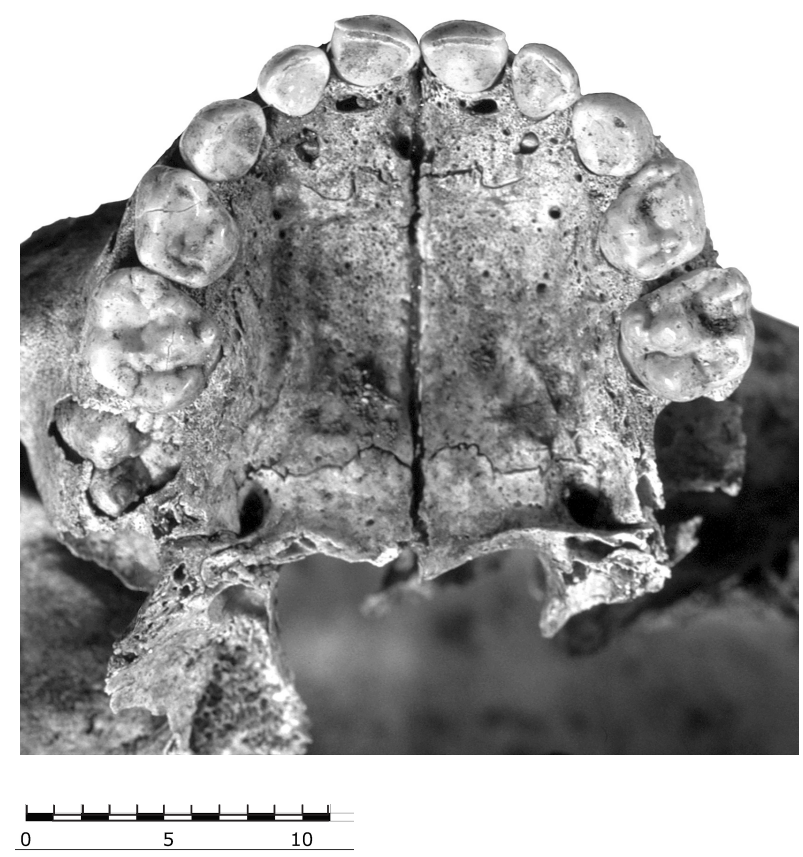

Abb. 4. Schwarzenbach-Burg, Grab 1, Detailaufnahme des Oberkiefers, Mahlzähne mit Tuberculi Carabelli.

viduen eine Körpergröße von ca. 85-94 cm und für subadulte weibliche ein Wert von 84-93 cm. Da das Geschlecht anthropologisch nicht sicher bestimmt werden konnte, wurde die Spanne gemittelt $(84-94 \mathrm{~cm})$.

\section{Maße des Schädels}

An dem postmortal stark verzogenen Kinderschädel können die angegebenen Maße nur mit Vorsicht für einen Vergleich herangezogen werden (Tab. 2).

\section{Morphologische Veränderungen}

Primär fällt an diesem Kinderschädel die ungewöhnlich stark nach oben gestreckte und in der Mitte eingezogene Stirn-
Tabelle 2. Maße des Schädels

\begin{tabular}{|l|l|l|}
\hline Martin Nr. & & $\mathrm{mm}$ \\
\hline 1. & Größte Hirnschädellänge & $(168)$ \\
\hline 2. & Glabello-Inionlänge & $(163)$ \\
\hline 3. & Glabello-Lambdalänge & 162 \\
\hline 8. & Größte Hirnschädelbreite & $(121)$ \\
\hline 9. & Kleinste Stirnbreite & 81 \\
\hline 23. & Horizontalumfang ü.d. Glabella & $(461)$ \\
\hline 25. & Mediansagittaler Bogen & $(332)$ \\
\hline 26. & Mediansagittaler Frontalbogen & 124 \\
\hline 27. & Mediansagittaler Parietalbogen & 118 \\
\hline 28. & Mediansagittaler Occipitalbogen & $(91)$ \\
\hline 29. & Mediansagittale Frontalsehne & 113 \\
\hline 30. & Mediansagittale Parietalsehne & 105 \\
\hline 31. & Mediansagittale Occipitalsehne & $(79)$ \\
\hline $32 / 1$. & Stirnneigungswinkel & 59 \\
\hline $32 / 2$. & Glabello-Bregma-Winkel & 52 \\
\hline
\end{tabular}

beinschuppe auf. Nach der Rekonstruktion des Schädels ist diese Eindellung auch an den Ossa parietalia zu erkennen. Es handelt sich um eine ringförmige, ca. $4 \mathrm{~cm}$ breite, flächige Einziehung, die im Stirnbein über den Tubera frontalia und im Scheitelbein unterhalb der Tubera parietalia liegt. Oberhalb dieser Einschnürung ist eine wulstartige Knochenerhebung deutlich zu erkennen. Eine zweite etwas schmälere Einziehung findet sich gleich nach der Bregmaregion, sie ist annähernd normal auf die oben beschriebene und zieht sich die Schläfenregion entlang hinunter. Die Veränderungen der Hinterhauptsschuppe sind bei der Projektion der Profilumrisse im Vergleich mit anderen, nicht deformierten Kinderschädeln (Abb. 5 und 6) deutlich erkennbar. So ist die Hinterhauptsschuppe in der Inionregion stark abgeflacht. Oberhalb des Lambdas ist eine weitere Einziehung zu erkennen. Der Schädel als Ganzes ist eindeutig höher als die zum Vergleich herangezogenen, nicht deformierten Kinderschädel aus Zwentendorf. An den abnehmbaren Schädelmaßen (Tab. 2) sind in der absoluten Knochengröße des Stirnbeines keine Verlängerungen messbar.

\section{Pathologische Veränderungen}

Im gesamten Schädeldach des Kindes sind deutlich verstärkte Abdrücke der Gehirnwindungen zu erkennen (Abb. 7). Weiters zeigen die Orbitadächer massive Cribra orbitalia mit sekundären Knochenauflagerungen. Die gesamte Kno- 
154
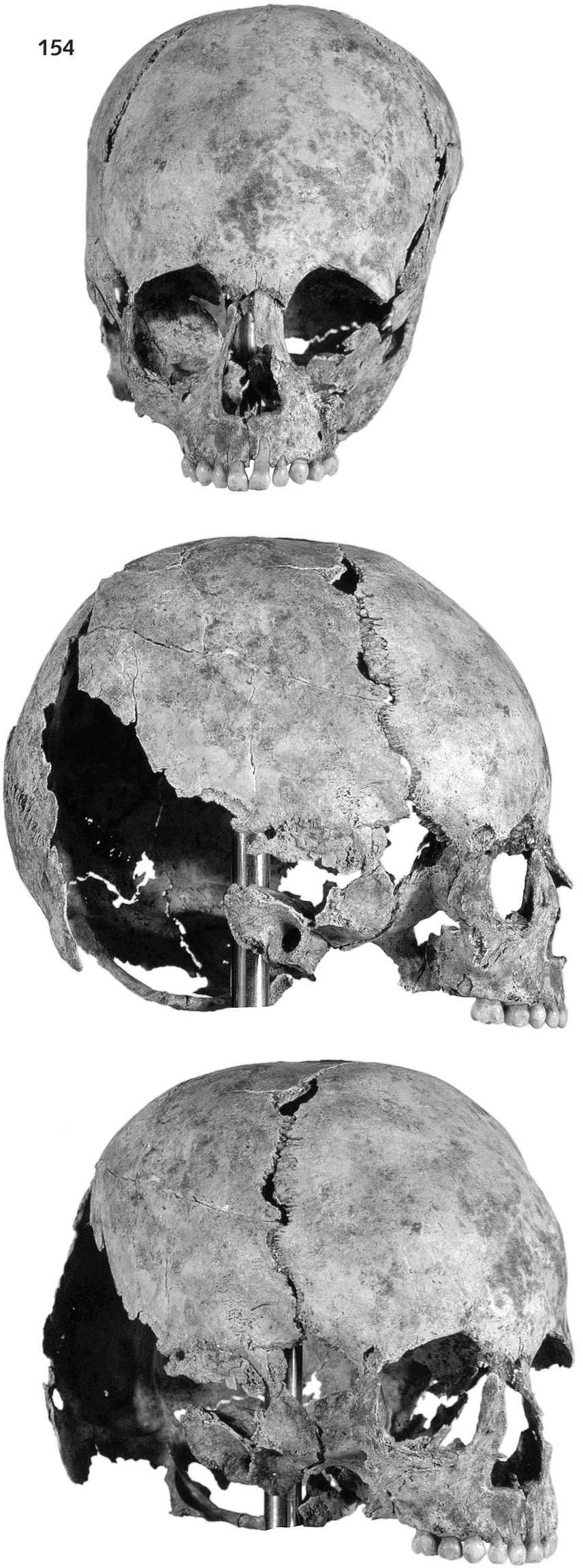
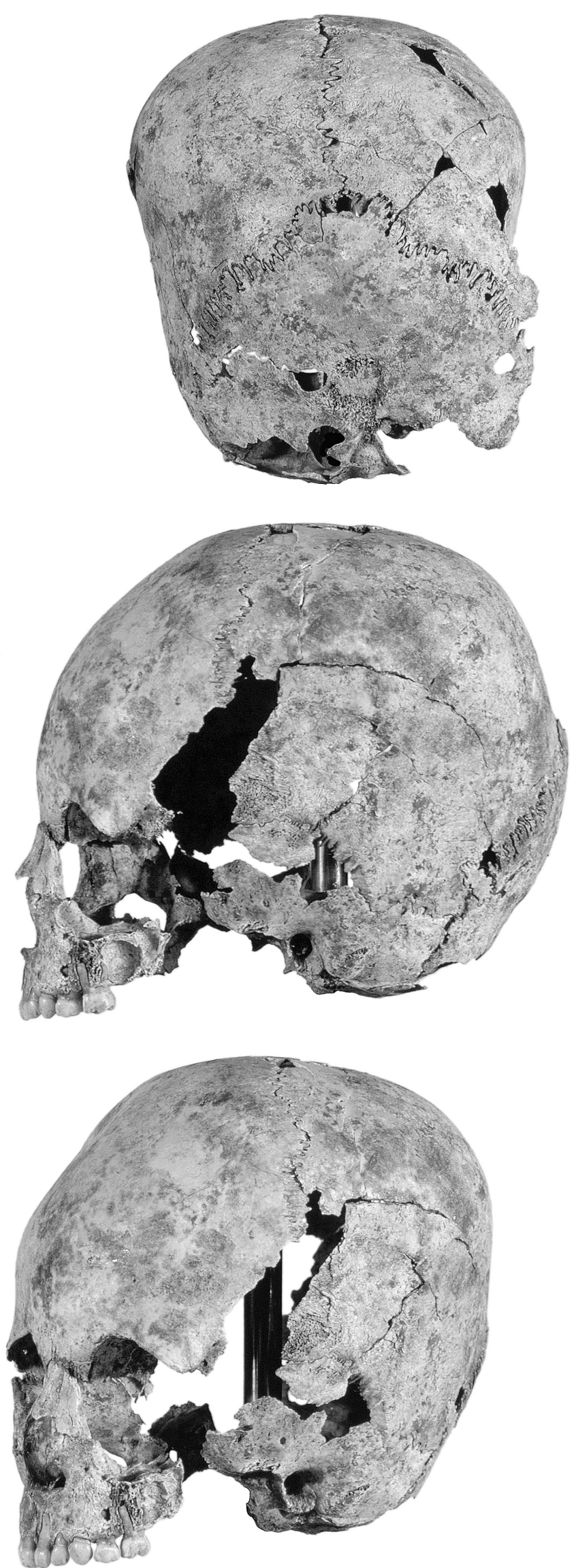

Abb. 5. Schwarzenbach-Burg, Grab 1, Normaufnahmen des künstlich deformierten Kinderschädels, Maßstab 1:2. 


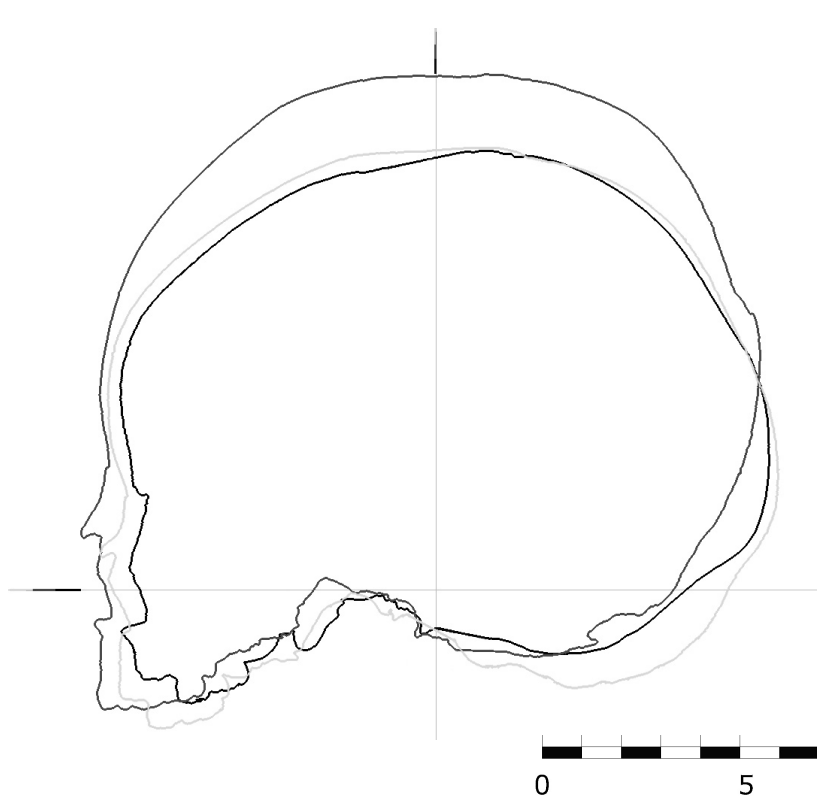

Abb. 6. Projektion der Schädelumrisse des künstlich deformierten Kinderschädels aus Schwarzenbach (dunkelgrau) mit den Umrisslinien der nicht deformierten Kinderschädel von Zwentendorf Grab 50 (ca. 3 Jahre, schwarz) und Grab 112/1 (ca. 5 Jahre, hellgrau).

Ausrichtung nach der Schädelbasis.

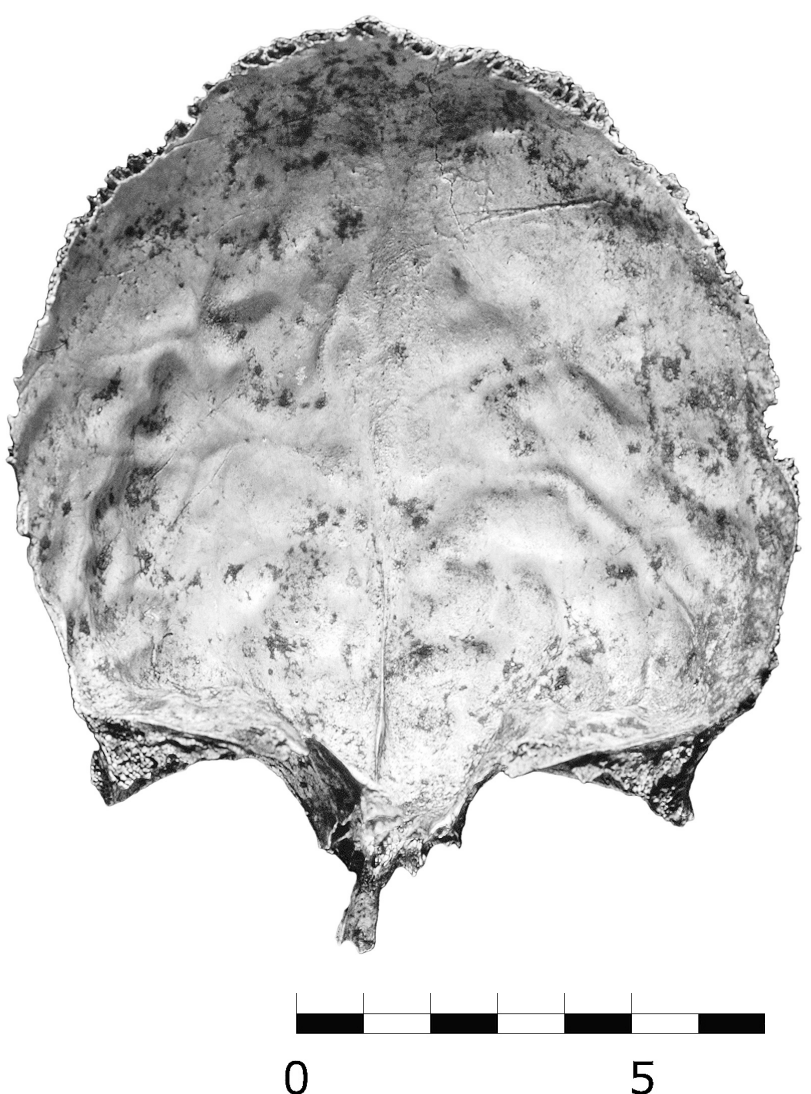

Abb. 7. Schwarzenbach-Burg, Grab 1, verstärkte Eindrücke der Gehirnwindungen (Impressiones digitatae) an der Schädelinnenseite des Stirnbeines. chenoberfläche des Oberkiefers ist feinporös und weist flächige Auflagerungen auf. Verstärkte Porositäten des äußeren Schädeldaches sind im Bereich der oberen Lambdanaht zu beobachten. Das rechte Scheitelbein zeigt in der vorderen Hälfte, knapp oberhalb der Naht zur Schläfenbeinschuppe eine Rauigkeit, die wie eine Gefäßeinsprossung aussieht. Diese Strukturen sind möglicherweise durch die Bodenlagerung verstärkt worden.

An allen Langknochen und an den Beckenschaufeln ist die Knochenoberfläche feinporös und weist sekundäre flächige Knochenauflagerungen auf, wobei die Schienbeinschäfte am stärksten betroffen sind (Abb. 8).

\section{Diagnose}

Die morphologischen Veränderungen am Kinderschädel deuten auf eine von außen erfolgte Manipulation im Sinne einer künstlichen Schädeldeformation hin. Die ringförmige, das Schädeldach umfassende, ca. $4 \mathrm{~cm}$ breite Eintiefung bzw.
Abflachung könnte durch eine Bandagierung des kindlichen Kopfes entstanden sein. Durch die daraus resultierende Druckausübung ist es neben der Verformung des Schädels wahrscheinlich auch zu einer Hirndrucksymptomatik gekommen, wodurch sich die Hirnwindungen besonders stark in der Lamina interna abdrückten.

Sowohl die Cribra orbitalia mit den starken sekundären Auflagerungen am Augendach, wie auch die an den Langknochen auffallenden Veränderungen deuten auf ein systemisches, pathologisches Geschehen hin. Die porösen, flächigen Knochenneubildungen am postkranialen Skelett können als Folge von verkalkten, subperiostalen Blutungen erklärt werden. Die Porositäten an den Knochenoberflächen weisen auf akute entzündliche Geschehen hin. Ähnliche Veränderungen können bei bestimmten Mangelerkrankungen wie z.B. bei Vitamin C-Mangel auftreten. Bei länger andauerndem Vitamin C-Mangel ist das Immunsystem geschwächt, und eine einfache Infektionskrankheit kann schnell zum Tod dieses Kleinkindes geführt haben. 


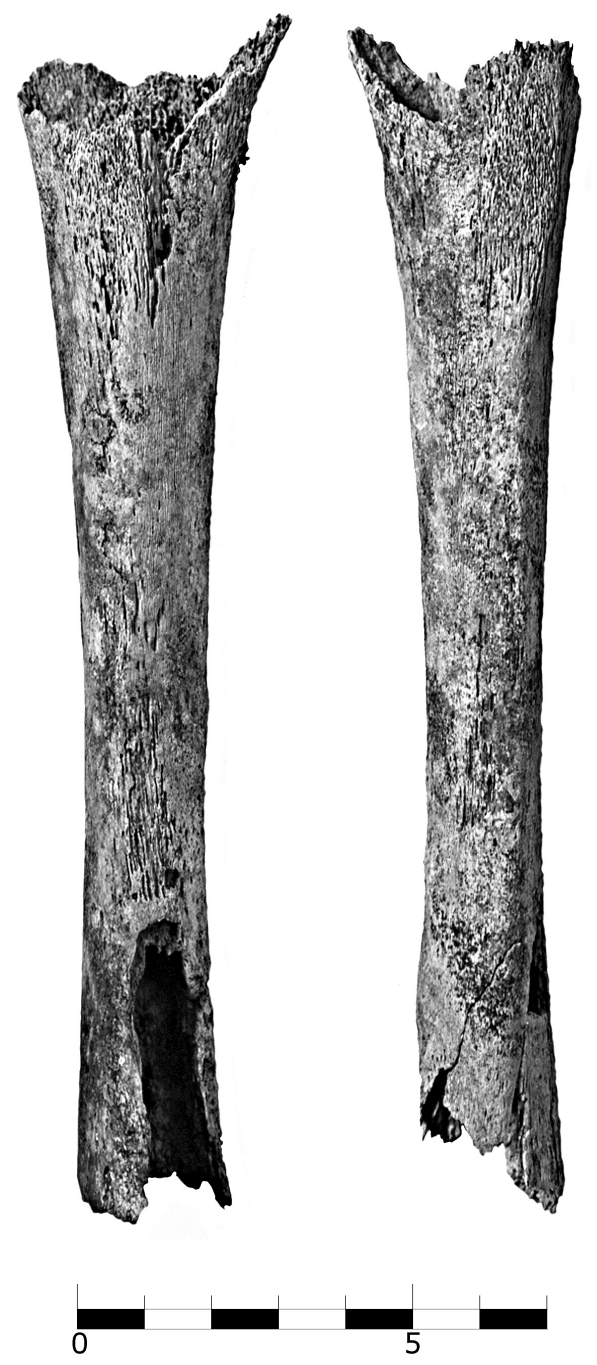

Abb. 8. Schwarzenbach-Burg, Grab 1, Schienbeinschäfte mit Porositäten und flächigen Knochenneuauflagerungen.

\section{Diskussion}

„Deformiert werden kann der kindliche Schädel durch verschiedene Methoden: durch starke Umschnürung mit Bändern, durch das Anbringen von Brettchen vor der Stirn

11 G.H.R. von KoenigSwald, Skelettkult der Vorgeschichte VI. Deformierte Schädel und Schrumpfköpfchen, Natur und Museum 109 (3), 1979, 65-69.

12 E.-M. WinkLer, J. Jungwirth, Ein Kinderskelett mit deformiertem Schädel aus Schiltern in Niederösterreich. Zur Geschichte und Technik der künstlichen Schädeldeformierung in Österreich, Fundber. aus Österr., 17, 1978, 197-209.

13 Winkler, Jungwirth, s. Anm. 12.

14 A. Schliz, Künstlich deformierte Schädel in germanischen Reihengräbern, Archiv für Anthropologie N.F. 3, 1905, 191-214.

15 E.J. Dingwall, Artificial Cranial Deformation, London 1931. und eventuell ein zweites Brettchen gegen den Hinterkopf gepresst. Es ist erstaunlich, was für Schädelformen sich durch Deformation erreichen lassen: überlange, überhohe, spitze und platte. Der Brauch findet sich in vielen Gebieten der Erde, und sehr verschiedene Motive haben dabei eine Rolle gespielt" ${ }^{\text {"11. }}$.

Der vorliegende Kinderschädel wurde wahrscheinlich in ähnlicher Weise wie das Kind von Schiltern mit zirkumferenten Binden deformiert ${ }^{12}$. Es ist allerdings nicht geklärt, ob die erkennbaren Einschnürungen, wie von E.-M. Winkler ${ }^{13}$ und A. Schlitz ${ }^{14}$ vorgeschlagen, durch ein einziges zirkumferentes Band entstanden sind, oder wie E. J. Dingwall ${ }^{15}$ und M. Ötzbek ${ }^{16}$ annehmen durch mindestens zwei Bänder, die annähernd normal aufeinander gelegt waren. M. Ötzbek beobachtete an seiner Serie von Kleinstkindern mit Schädeldeformationen aus dem türkischen Chalkolithikum, dass bei Kindern bis zum 1. Lebensjahr die präbregmatische Abflachung des Stirnbeines durch ein einziges Band hervorgerufen wurde. Die postbregmatische Einziehung ist erst bei Kindern, die älter als ein Jahr sind, zu sehen. Er nimmt an, dass diese Deformation erst nach dem ersten Lebensjahr durch ein zweites Band, welches möglicherweise vom Bregma zum Kinn gebunden wurde, entstand. Dass in unterschiedlichem Alter verschiedene Methoden der Schädeldeformation angewandt werden, beschreibt E. J. Dingwall ${ }^{17}$ an Bräuchen im 19. Jh. in Frankreich. So war es in Deux-Sèvres üblich, dem Neugeborenen bis zum 2.-4. Monat ein einziges Band anzulegen. Danach wurde dem Kleinkind eine harte Kappe aus Pappe? (Calotte) aufgesetzt und später eine mit Draht versetzte Kopfbedeckung (z.B. Bèguins) verwendet, um die Form des Kopfwachstums zu beeinflussen. Nach O. Röhrer-Ertl und K.-W. Frey ${ }^{18}$ hatte diese Bindung keine Auswirkung auf die Sinnesleistungen des Menschen. O. Vyslozil und R. Slavicek ${ }^{19}$ stellten auch fest, dass sich die Okklusion des Kauapparates an die veränderten Druckverhältnisse anpasste.

Welche mechanischen Kräfte auch eingewirkt haben mögen, einige der Kinder mit Schädeldeformationen sind auch erwachsen geworden und die ausgeprägten Verfor-

16 M. Ötzbeк, Cranial Deformation in a Subadult Sample From Degirmentepe (Chalcolithic, Turkey), Am. J. Phys. Anthrop. 115, 2001, 238-244.

17 Dingwall, s. Anm. 15, 50.

18 O. RÖHrer-Ertl, K.-W. Frey, Über den Einfluß der künstlichen Schädeldeformation in vivo auf die Sinnesleistungen beim Menschen, Gegenbauers morph. Jahrb. Leipzig 130/5, 1984, 677697.

19 O. Vyslozil, R. Slavicek, Vergleichsuntersuchungen an künstlich deformierten und undeformierten Schädeln, Ann. Naturhist. Mus. Wien 102A, 2001, 245-274. 


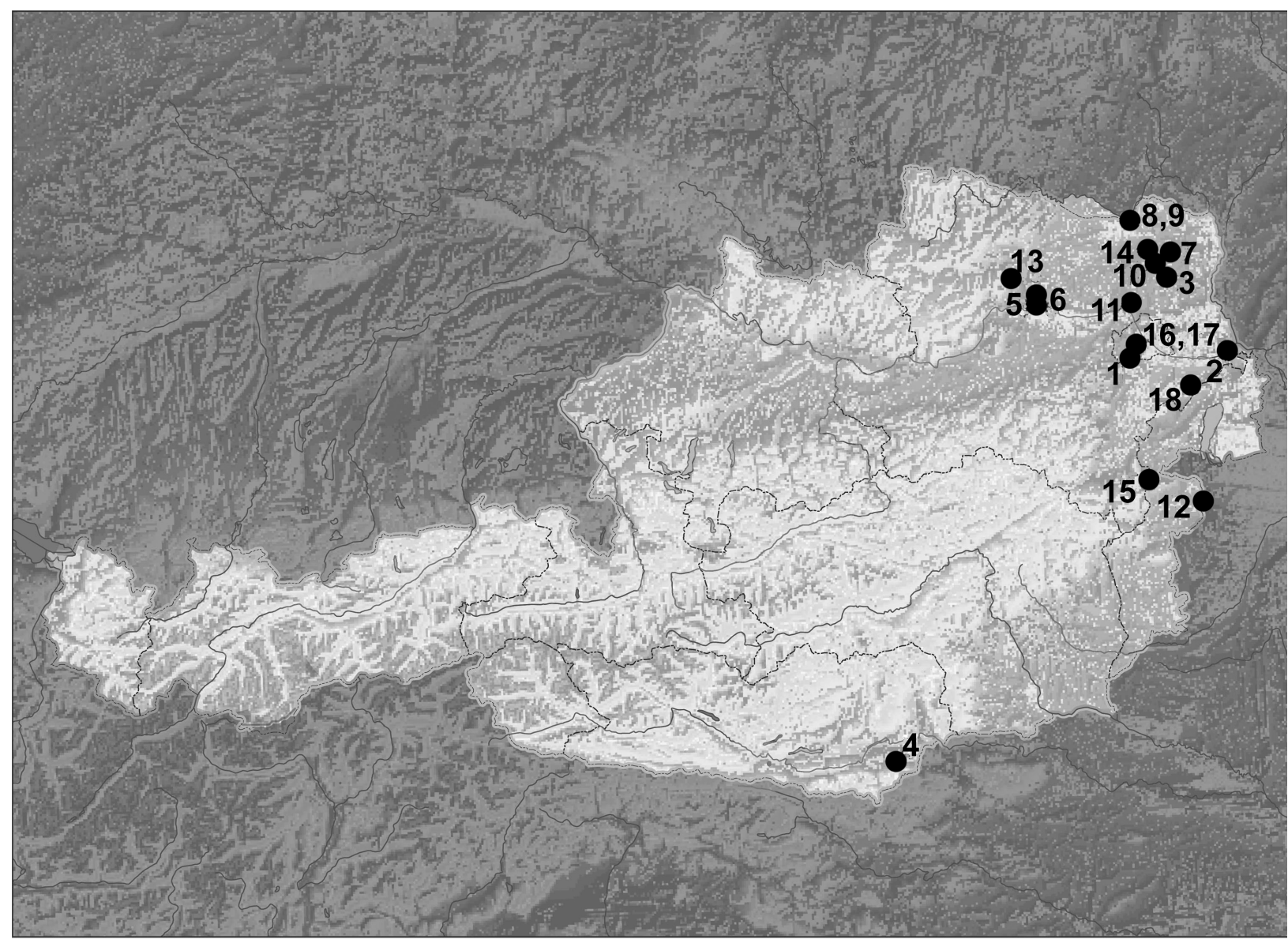

Abb. 9. Fundstellen mit deformierten Schädeln in Österreich. 1 Atzgersdorf, 2 Bad Deutsch-Altenburg, 3 Gaweinstal, 4 Globasnitz, 5 Grafenegg, 6 Grafenwörth, 7 Hobersdorf, 8-9 Laa an der Thaya, 10 Ladendorf, 11 Leobendorf, 12 Nikitsch, 13 Schiltern, 14 Schletz, 15 Schwarzenbach, 16 Wien Salvatorgasse, 17 Wien Mariahilfergürtel, 18 Sommerein.

mungen des Hirnschädels blieben ein Leben lang erhalten. In Österreich sind bis jetzt 18 Fundstellen mit künstlich deformierten Schädeln bekannt (Abb. $9^{20}$ ). Bei sechs der in Österreich bislang gefundenen Skelette mit deformierten Schädeln handelt es sich um Kinder oder Jugendliche. Diese stammen aus Laa- Mitterhof (ca 13 Jahre ${ }^{21}$ ), Schiltern (ca. 5 Jahre $^{22}$ ), Leobendorf (Infans $\left.\mathrm{I}^{23}\right)$, Gaweinstal (9-10 Jahre $\left.^{24}\right)$, Hobersdorf (juveni1 ${ }^{25}$ ) und Schwarzenbach (3-4 Jahre ${ }^{26}$ ).

20 Herzlichen Dank an Mag. Bence Viola, der die Kartierung durchgeführt hat.

21 M. Teschler-Nicola, Die künstlich deformierten Schädel aus Laa an der Thaya, Mitterhof, in Arbeit.

22 WinkLer, Jungwirth, s. Anm. 12.

23 E.-M. Winkler, L. Wicke, Hunnenzeitliche Skelettfunde mit künstlich deformierten Schädeln aus Gaweinstal in Niederösterreich, ArchA 64, 1980, 119-137, Abb. 5.
Manche dieser doch recht auffälligen Schädel sind schon im 19. Jh. wissenschaftlich beschrieben worden ${ }^{27}$. Augenscheinlich sind alle mit einer ähnlichen Technik, durch im Säuglingsalter fest angelegte Bandagen oder Bänder, verformt worden ${ }^{28}$.

Für den österreichischen Raum wird angenommen, dass die Hunnen und die von ihnen beeinflussten Sarmaten, die Technik der Schädeldeformation aus Zentralasien nach
24 WinkLer, Wicke, s. Anm. 23

25 Unbearbeitet, Bestimmung durch die Autorin.

26 K. WiltschKe, D. PANY, vorliegende Arbeit.

27 L.J. Fitzinger, Die Schädel der Avaren insbesondere über die seither in Österreich aufgefundenen, Denkschr. mathem.-naturwiss. Cl. kaiserl. Akad. d. Wiss. 5, 1853, 3-16.

28 Fitzinger, s. Anm. 27. -Schliz, s. Anm. 14. - Winkler, Wicke, s. Anm. 23. 
Mitteleuropa gebracht haben.Viele germanische Völker, die unter die Herrschaft der Hunnen gerieten, wie die Gepiden, die Ostgoten, die Rugier, die Heruler und die Burgunder dürften diesen Brauch in der 1. Hälfte des 5. Jhs. übernommen haben. Bei westgermanischen Stämmen wie den Quaden, den Thüringern und den Langobarden sind künstliche Schädeldeformationen nur sehr vereinzelt zu finden. Die Sitte der künstlichen Schädeldeformation im 5. Jh. war ein Kulturelement, das im Zuge politischer Vorgänge mit einer von Reiternomaden getragenen kurzfristigen Machtzusammenballung weit nach Westen getragen wurde und schon bald nach dem Zusammenbrechen des hunnischen Großreiches wieder aufgegeben wurde ${ }^{29}$. Das Deformieren kindlicher Schädel mag in Europa eine „Modeerscheinung“ dieser Zeit gewesen sein, die sicher nicht nur auf die Form des Schädels beschränkt war, sondern auch Kleidung, Schmuck und Bewaffnung umfasste ${ }^{30}$.

Deformierte Schädel können auch schon in proto-neolithischen und neolithischen Funden aus der Shanidar Höhle im Irak nachgewiesen werden ${ }^{31}$. Aber auch in einem mittelalterlichen Gräberfeld in der Slowakei konnten künstlich deformierte Schädel festgestellt werden ${ }^{32}$. E.J. Dingwall ${ }^{33}$ beschreibt ausführlich die weltweit zu unterschiedlichsten Zeiten üblichen Sitten, Traditionen und die unterschiedlichsten Arten der Schädeldeformationen. Noch im 19. Jh. war es in einigen Distrikten Frankreichs Brauch, die Schädel der Kinder zunächst mit Bändern und später mit drahtverstärkten Hauben ${ }^{34}$ zu deformieren.

Die Intention der Mutter oder der Kindesbetreuerin, den Schädel eines Kindes zu verformen setzt grundsätzliche Überlegungen voraus. Der Aufwand dafür war sicherlich sehr groß. So wird in völkerkundlichen Beobachtungen beschrieben, dass die Mütter die straffen Bandagen immer wieder abgenommen haben und neu aufsetzten ${ }^{35}$. E.J. Dingwall ${ }^{36}$ dagegen zitiert F. Delisle's ${ }^{37}$ Beobachtungen der Schä- deldeformation in Frankreich im 19.Jh.: „Among the pieces of apparatus used were handkerchiefs, the Bandeau and occasionally a band of cloth $4 \mathrm{~cm}$ to $5 \mathrm{~cm}$ wide and $2 \mathrm{~m}$ or more in length which was tightly wound round the head. The child often cried for hours under the application of these constrictions without the parents realizing the cause of the infants' distress. The bandage was rarely removed and consequently harboured thousands of lice which contributed to the development of skin affections and suppurating ulcers."

Ein künstlich deformierter Schädel hat nicht nur eine ästhetische Komponente, er prägt das Individuum auch in seiner Identität. Der Schädel wird zum fundamentalen Teil einer Persönlichkeit. Es ist zudem ein ausdrucksvolles, ständig sichtbares, deutliches Symbol einer sozialen Zugehörigkeit $^{38}$.

Da zur Zeit noch keine weiteren völkerwanderungszeitlichen Funde in der Gegend um Schwarzenbach vorliegen, kann man eventuell auch eine Bestattung des Kindes auf der „Durchreise“ in Erwägung ziehen. Eine Bestattung in einem Holzsarg mit Steinabdeckung erscheint wahrscheinlich. Das Grab dürfte kurz nach der Bestattung beraubt worden sein. Die Schädeldeformation, die Lage der in Stein gehauenen Grabgrube an der höchsten Stelle des Hügels - einem sehr markanten Punkt in der Umgebung - lassen bei dem Kind, trotz fehlender Grabbeigaben, eine soziale Sonderstellung vermuten.

\section{Nachtrag:}

In Mannersdorf am Leithagebirge wurde 2003 am Sandberg beim Sandabbau ein Skelett eines 25-35jährigen Mannes mit einer künstlichen Schädeldeformation ausgebaggert. Die Grabsituation war nicht mehr rekonstruierbar. Das Skelett wird nach der wissenschaftlichen Bearbeitung im Museum Mannersdorf ausgestellt.
29 J. Werner, Beiträge zur Archäologie des Attila-Reiches. Die artifizielle Schädeldeformation, Bayr. Akad. d. Wiss. Phil.-Hist. Kl. Abh. N.F. 38A, 1956, 5-18, 96-114.

30 H. Friesinger, B. Vacha, Die vielen Väter Österreichs. RömerGermanen-Slawen. Eine Spurensuche, Wien 1987, 67-68.

31 C. Meikrejohn et al., Artificial cranial deformation in the protoneolithic and neolithic Near East and its possible origin: Evidence from four sites, Paléorient 18/2, 1992, 83-97.

32 R. Benus, S. Masnicová, J. Lietava, Intentional Cranial Vault Deformation in a Slavonic Population from the Medieval Cemetary in Devín (Slovakia), Internat. Journ. of Osteoarchaeology 9, 1999, 267-270.
33 Dingwall, s. Anm. 15.

34 Dingwall, s. Anm. 15, 47-48.

35 G. Buschan, Die Verunstaltung des kindlichen Körpers in der Völkerkunde, Kinderärztliche Praxis 6/2, 1935, 73-81.

36 Dingwall, s. Anm. 15.

37 F. Delisle, Les deformations artificielles du crane en France.

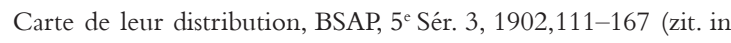
Dingwall, s. Anm. 15).

38 C. Torres-Rouff, Cranial Vault Modification and Ethnicity in Middle Horizon San Pedro de Atacama, Chile, Current Anthropology 43/1, 2002, 163-171. 
Tabelle 3. Zusammenstellung der in Österreich gefundenen künstlich deformierten Schädel mit wichtigsten Literaturdaten und den derzeitigen Aufbewahrungsorten ${ }^{39}$.

\begin{tabular}{|c|c|c|c|c|c|}
\hline Ort & $\begin{array}{l}\text { weitere Orts- } \\
\text { bezeichnungen }\end{array}$ & Zeitstellung & Fundjahr & Individuum & Verbleib \\
\hline Atzgersdorf ${ }^{40}$ & $\begin{array}{l}\text { Inzersdorf, Wien } \\
\text { aus Steinbruch }\end{array}$ & 5. Jh. & 1846 & Frau, ca. 20 Jahre & $\begin{array}{l}\text { Path.Anat Mus. MN:16.910/ } \\
\text { 1182a } \\
\text { Abguss, 16.910/1183 }\end{array}$ \\
\hline $\begin{array}{l}\text { Bad Deutsch- } \\
\text { Altenburg }^{42}\end{array}$ & Carnuntum & nicht datierbar & & 1 & $\begin{array}{l}\text { ehem. Besitz C. Toldt } \\
\text { Verbleib?? }\end{array}$ \\
\hline Gaweinstal $^{43}$ & $\begin{array}{l}\text { Kollnbrunn } \\
\text { BH Mistelbach } \\
\text { Parz. 2124/1 }\end{array}$ & Mitte 5. Jh. & $\begin{array}{l}1975 / \\
76\end{array}$ & $\begin{array}{l}\text { S1: Mann, adult, deformiert } \\
\text { S2: Frau, matur, deformiert } \\
\text { S3: Frau, adult, deformiert } \\
\text { S4: Infans II, deformiert } \\
\text { S5: Mann, juvenil, defor- } \\
\text { miert } \\
\text { S6: Frau, frühad., deformiert } \\
\text { (S7, S8, S9: kein Schädel } \\
\text { vorhanden) }\end{array}$ & $\begin{array}{l}\text { Privatbesitz H.Bauch } \\
\text { S4 Institut für Anthropologie } \\
\text { Univ. Wien }\end{array}$ \\
\hline Globasnitz $^{44}$ & $\begin{array}{l}\text { BZ Völkermarkt } \\
\text { Hemmaberg }\end{array}$ & 5.-6. Jh. & $\begin{array}{l}2000 / \\
2002 \\
\end{array}$ & $\begin{array}{l}\text { derzeit } 5 \text { deformierte } \\
\text { Schädel }\end{array}$ & $\begin{array}{l}\text { Landesmuseum Kärnten, } \\
\text { Dr. Glaser }\end{array}$ \\
\hline Grafenegg $^{45}$ & Feuersbrunn & & 1820 & 1 Frau, erwachsen & $\begin{array}{l}\text { Original verschollen, } \\
\text { Gipsabguss in Anthrop. } \\
\text { Abt. NHM } \\
\text { Invnr. } 5438\end{array}$ \\
\hline Grafenwörth $^{46}$ & $\begin{array}{l}\text { Schottergrube } \\
\text { Holzer, Parz. } 337 \\
\text { Ried "an den See" } \\
\text { BH Tulln }\end{array}$ & $\begin{array}{l}420-468 \\
\text { rugisch }\end{array}$ & $\begin{array}{l}1922 \\
1939 \\
1940\end{array}$ & $\begin{array}{l}1 \text { Frau } \\
1 \text { Mann } \\
\text { aus } 18 \text { Steinplatten- } \\
\text { gräbern }\end{array}$ & $\begin{array}{l}\text { Bezirksmuseum Tulln } \\
\text { Invnr. } 1179\end{array}$ \\
\hline Hobersdorf ${ }^{47}$ & $\begin{array}{l}\text { BH Mistelbach } \\
\text { Schottergrube } \\
\text { Döltl }\end{array}$ & $\begin{array}{l}\text { hunnenzeitlich } \\
\text { 5. Jh. }\end{array}$ & 1950 & $\begin{array}{l}1 \text { adult, männlich } \\
2 \text { weitere unveröffentlicht }\end{array}$ & $\begin{array}{l}\text { Anthrop. Abt. NHM } \\
\text { Invnr. 21187, 21203, } 21204\end{array}$ \\
\hline Laa/Thaya ${ }^{48}$ & $\begin{array}{l}\text { BH Mistelbach, } \\
\text { Alte Ziegelei } \\
\text { östlich vom } \\
\text { Bahnhof }\end{array}$ & $\begin{array}{l}\text { 1. Hälfte } 5 . \text { Jh. } \\
\text { gotisch }\end{array}$ & $\begin{array}{l}1908 / \\
1909\end{array}$ & 1 Frau & $\begin{array}{l}\text { Anthrop. Abt. NHM } \\
\text { Invnr. } 5460\end{array}$ \\
\hline
\end{tabular}

39 Basiert teilweise auf Angaben von H. WindL et al., Germanen, Awaren, Slawen in Niederösterreich. Das erste Jahrtausend nach Christus. Ausstellungskatalog Niederösterr. Landesmus., Wien 1977. - Werner, s. Anm. 29.

40 E.-M. WinkLER, Der künstlich deformierte Schädel von Atzgersdorf - Fragen seiner Herkunft und Bedeutung aus heutiger Sicht, Mitt. Anthrop. Ges. Wien 109, 1979, 1-9. - Werner, s. Anm. 29, 109. - Schliz, s. Anm. 14, 207. - Dingwall, s. Anm. 15, 26.

41 K.A. Portele, Die Sammlung mazerierter Skelette und Knochen des Pathologisch-anatomischen Bundesmuseums in Wien, Teil 1, Mitt. Patholog.-anatom. Bundesmus. Wien, N.F. 1, 1982, 206.

42 Schliz, s. Anm. 14, 191-214. - Werner, s. Anm. 29, 110.

43 Winkler, Wicke, s. Anm. 23.

44 F. GLaser, Künstliche Schädeldeformation in Kärnten, ArchA 84-85, 2000-2001, 291-294.
45 Fitzinger, s. Anm. 27. - Werner, s. Anm. 29, 110. - Schliz, s. Anm. 14, 206f. - Dingwall, s. Anm. 15, 26.

46 A. Lippert, Ein Gräberfeld derVölkerwanderungszeit bei Grafenwörth, p.B. Tulln, NÖ, Mitt. Anthrop. Ges. Wien 98, 1968, 35 ff. - Fundber. Österr. 3, 1948, 167; Fundber. Österr. 4, 1952, 68. Werner, s. Anm. 29, 110. Verbleib: Laut allg. Korr. Nr. P204/68 der Anthrop. Abt. in Verlust geraten, Unterkiefer Anthrop. Abt. Invnr. 22058 in osteol. Sammlung vorhanden.

47 W. Ehgartner, Zur Anthropologie des Langobardenschädels von Hobersdorf, NÖ. In: H. Mitscha-Märheim, Neue Bodenfunde zur Geschichte der Langobarden und Slawen im österreichischen Donauraum, Carinthia 1, 1953,359-363. - Werner, s. Anm. 29, 110 .

48 E. Beninger, Germanengräber von Laa an der Thaya, NÖ, Eiszeit und Urgeschichte 6, 1929, 143-155. - Werner, s. Anm. 29, 110. 
Tabelle 3 (Ende)

\begin{tabular}{|c|c|c|c|c|c|}
\hline Ort & $\begin{array}{l}\text { weitere Orts- } \\
\text { bezeichnungen }\end{array}$ & Zeitstellung & Fundjahr & Individuum & Verbleib \\
\hline Laa/Thaya ${ }^{49}$ & $\begin{array}{l}\text { Mitterhof, } \\
\text { Wildendürnbach, } \\
\text { Große Steinbreite } \\
\text { BH Mistelbach }\end{array}$ & 1. Hälfte $5 . \mathrm{Jh}$. & $\begin{array}{l}1974 / \\
1975\end{array}$ & $\begin{array}{l}\text { Grab 3: Frau, juvenil, } \\
\text { deformiert } \\
\text { Grab 5: Frau, adult, } \\
\text { deformiert } \\
\text { Grab 11: Mann, } \\
\text { adult-matur, deformiert }\end{array}$ & $\begin{array}{l}\text { Anthrop. Abt. NHM } \\
\text { zur Bearbeitung } \\
\text { M. Teschler-Nicola }\end{array}$ \\
\hline Ladendorf $^{50}$ & $\begin{array}{l}\text { MG Ladendorf } \\
\text { BH Mistelbach }\end{array}$ & $\begin{array}{l}\text { 5. Jh. } \\
\text { frühmerowinger- } \\
\text { zeitlich }\end{array}$ & 1995 & 1 Frau & $\begin{array}{l}\text { Frühgeschichtsmuseum } \\
\text { Traismauer, ausgestellt }\end{array}$ \\
\hline Leobendorf ${ }^{51}$ & $\begin{array}{l}\text { Sandgrube der } \\
\text { Gemeinde, } \\
\text { BH Korneuburg }\end{array}$ & & 1958 & 1 Kind & $\begin{array}{l}\text { Städtisches Museum der } \\
\text { Stadtgemeinde Korneuburg, } \\
\text { ausgestellt }\end{array}$ \\
\hline Nikitsch $^{52}$ & BH Oberpullendorf & $\begin{array}{l}\text { ab 2. Hälfte } 6 . \mathrm{Jh} \text {. } \\
\text { langobardisch }\end{array}$ & 1930 & 1 Frau & $\begin{array}{l}\text { Anthrop. Abt. NHM } \\
\text { Invnr. } 6053\end{array}$ \\
\hline Schiltern $^{53}$ & $\begin{array}{l}\text { Burgstall, Gem. } \\
\text { Langenlois, BH } \\
\text { Krems/Donau }\end{array}$ & & 1939 & 1 Kind 5-6 Jahre & $\begin{array}{l}\text { Frühgeschichtsmuseum } \\
\text { Traismauer NL19013 }\end{array}$ \\
\hline Schletz $^{54}$ & $\begin{array}{l}\text { MG Asparn/Zaya } \\
\text { BH Mistelbach }\end{array}$ & $\begin{array}{l}\text { 5. Jh. } \\
\text { herulisch }\end{array}$ & 1993 & 1 Erwachsener & $\begin{array}{l}\text { Anthrop. Abt. NHM } \\
\text { zur Bearbeitung } \\
\text { M. Teschler-Nicola }\end{array}$ \\
\hline $\begin{array}{l}\text { Schwarzen- } \\
\text { bach }\end{array}$ & $\begin{array}{l}\text { BH Wiener- } \\
\text { Neustadt }\end{array}$ & $380-430$ & 1998 & Infans I, 3-4 Jahre & Turmmuseum Schwarzenbach \\
\hline Sommerein $^{55}$ & $\begin{array}{l}\text { Flur Steinäcker Parz. } \\
6567 / 1\end{array}$ & $435-455$ & 1985 & Grab 261, Frau, frühadult & Anthrop. Abt. NHM \\
\hline $\begin{array}{l}\text { Wien } 1, \\
\text { Salvatorgasse }{ }^{56}\end{array}$ & Wien & $\begin{array}{l}\text { Ende 5. Anfang } \\
\text { 6. Jh. } \\
\text { langobardisch }\end{array}$ & 1951 & $\begin{array}{l}1 \text { Mann (Grab 3) sicher } \\
2 \text { Indiv. ? } \\
1 \text { Frau nicht deformiert }\end{array}$ & verschollen \\
\hline $\begin{array}{l}\text { Wien 6, } \\
\text { Mariahilfer- } \\
\text { gürtel }^{57}\end{array}$ & Wien, Mittelgasse & $\begin{array}{l}\text { zweites Drittel } \\
6 . \text { Jh. }\end{array}$ & $\begin{array}{l}1897 / \\
1898\end{array}$ & 1 aus 20 & $\begin{array}{l}\text { Historisches Museum der } \\
\text { Stadt Wien I.N. 8686, MV/55, } \\
\text { LXXIX-8 1897, ausgestellt }\end{array}$ \\
\hline
\end{tabular}

49 H. Dick, A. Sedlarik, Fundber. Österr. 13, 1974, 135. - A. Sedlarik, Fundber. Österr. 14, 1975, 178. - A. Heinrich, Ein völkerwanderungszeitliches Gräberfeld bei Mitterhof, GB Laa an der Thaya, Niederösterreich, ArchA 74, 1990, 85-104. - Teschler-NicOLA, s. Anm. 21.

50 H. WindL, Fundber. Österr. 34, 1995, 731.

51 H. Göttinger, N. ZeissL, Fundber. Österr. 7, 1956, 150. - G. MossLER, Österr. Zeitschr. f. Kunst und Denkmalpflege 12, 1958, 110.

52 G. Müller, Zur Anthropologie der Langobarden, Mitt. Anthrop. Ges. Wien 66, 1936, 344-355. - Werner, s. Anm. 29, 110.

53 Winkler, Jungwirth, s. Anm. 12.
54 H. WINDL, Fundber. Österr. 32, 1993, 678, 777.

55 J. Szilvassy, H. Kritscher, Diagnose nach 1000 Jahren. Katalog zur Sonderausstellung des Burgenländischen Landesmuseums NF 30, Eisenstadt, 1988, 51, Abb. 78. - F. DAim, Fundber. Österr. 24/25, 1985/1986, 334, hier irrtüml. als Grab 161 bezeichnet.

56 H.-M. PACHER, Die langobardischen Gräber - Anhang: Anthropologischer Befund von S1-S4. In: A. Neumann, Spital und Bad des Legionslagers Vindobona, Jahrb. Röm.-German. Zentralmus. Mainz 12, 1965, 117-126. - Werner, s. Anm. 29, 110.

57 M. Much, Correspondenzblatt d. Deutschen Anthrop. Ges. 12, 1898 - Schliz, s. Anm. 14. - Werner, s. Anm. 29, 110. 